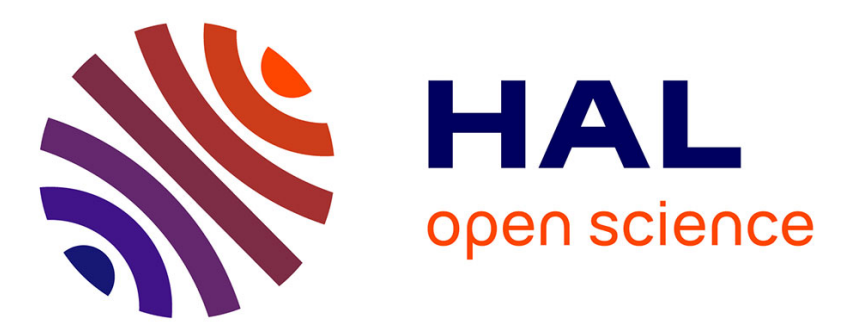

\title{
Offset and anteversion reconstruction after cemented and uncemented total hip arthroplasty: an evaluation with the low-dose EOS system comparing two- and three-dimensional imaging
}

Jean-Yves Lazennec, Adrien Brusson, Dominique Folinais, Marc-Antoine

Rousseau, Aidin Eslam Pour

\section{To cite this version:}

Jean-Yves Lazennec, Adrien Brusson, Dominique Folinais, Marc-Antoine Rousseau, Aidin Eslam Pour. Offset and anteversion reconstruction after cemented and uncemented total hip arthroplasty: an evaluation with the low-dose EOS system comparing two- and three-dimensional imaging. International Orthopaedics, 2015, 39, pp.1259-1267. 10.1007/s00264-014-2616-3 . hal-02483318

\author{
HAL Id: hal-02483318 \\ https://hal.science/hal-02483318
}

Submitted on 18 Feb 2020

HAL is a multi-disciplinary open access archive for the deposit and dissemination of scientific research documents, whether they are published or not. The documents may come from teaching and research institutions in France or abroad, or from public or private research centers.
L'archive ouverte pluridisciplinaire HAL, est destinée au dépôt et à la diffusion de documents scientifiques de niveau recherche, publiés ou non, émanant des établissements d'enseignement et de recherche français ou étrangers, des laboratoires publics ou privés. 


\title{
Offset and anteversion reconstruction after cemented and uncemented total hip arthroplasty: an evaluation with the low-dose EOS system comparing two- and three-dimensional imaging
}

\author{
Jean Yves Lazennec • Adrien Brusson • Folinais Dominique • \\ Marc-Antoine Rousseau • Aidin Eslam Pour
}

\begin{abstract}
Purpose Accurate evaluation of femoral offset is difficult with conventional anteroposterior (AP) X-rays. The EOS imaging system is a system that makes the acquisition of simultaneous and orthogonal AP and lateral images of the patient in the standing position possible. These twodimensional (2D) images are equivalent to standard plane Xrays. Three-dimensional $(3 \mathrm{D})$ reconstructions are obtained from these paired images according to a validated protocol. This prospective study explores the value of the EOS imaging system for comparing measurements of femoral offset from these $2 \mathrm{D}$ images and the $3 \mathrm{D}$ reconstructions.

Methods We included 110 patients with unilateral total hip arthroplasty (THA). The 2D offset was measured on the AP
\end{abstract}

\footnotetext{
J. Y. Lazennec

Department of Orthopaedic and Trauma Surgery, Pitié-Salpétrière Hospital Assistance Publique-Hopitaux de Paris, UPMC, 47-83 boulevard de l'hôpital, 75013 Paris, France

J. Y. Lazennec $\cdot$ M.-A. Rousseau

Biomechanics Lab (LBM), Arts et Metiers Paris-Tech, Paris, France

J. Y. Lazennec • A. Brusson • M.-A. Rousseau

Department of Anatomy, UPMC, 105 boulevard de l'hôpital,

75013 Paris, France

M.-A. Rousseau

Department of Orthopaedic and Trauma Surgery, Avicenne Hospital, Assistance Publique-Hopitaux de Paris, 125 rue de Stalingrad, 93009 Bobigny, France

F. Dominique

RIM Maussins-Nollet, 114 rue Nollet, 75017 Paris, France

A. E. Pour $(\bowtie)$

Department of Orthopaedic Surgery, University of Michigan, Ann Arbor, MI, USA

e-mail: aeslampo@med.umich.edu
}

view with the same protocol as for standard X-rays. The 3D offset was calculated from the reconstructions based on the orthogonal AP and lateral views. Reproducibility and repeatability studies were conducted for each measurement. We compared the 2D and 3D offset for both hips (with and without THA).

Results For the global series (110 hips with and 110 without THA), 2D offset was $40 \mathrm{~mm}$ (SD 7.3; 7-57 mm). The standard deviation was $6.5 \mathrm{~mm}$ for repeatability and $7.5 \mathrm{~mm}$ for reproducibility. Three-dimensional offset was $43 \mathrm{~mm}$ (SD 6.6; 22-62 mm), with a standard deviation of 4.6 for repeatability and 5.5 for reproducibility. Two-dimensional offset for the hips without THA was $40 \mathrm{~mm}$ (SD 7.0; 26-56 mm), and 3D offset $43 \mathrm{~mm}$ (SD 6.6; 28-62 mm). For THA side, 2D offset was $41 \mathrm{~mm}$ (SD 8.2; 7-57 mm) and 3D offset $45 \mathrm{~mm}$ (SD 4.8; 22-61 mm). Comparison of the two protocols shows a significant difference between the 2D and 3D measurements, with the $3 \mathrm{D}$ offset having higher values. Comparison of the side with and without surgery for each case showed a 5-mm deficit for the offset in $35 \%$ of the patients according to the $2 \mathrm{D}$ measurement but in only $26 \%$ according to the $3 \mathrm{D}$ calculation.

Conclusions This study points out the limitations of 2D measurements of femoral offset on standard plane Xrays. The reliability of the EOS 3D models has been previously demonstrated with $\mathrm{CT}$ scan reconstructions as a reference. The EOS imaging system could be an option for obtaining accurate and reliable offset measurements while significantly limiting the patient's exposure to radiation.

Keywords Total hip arthroplasty · Offset and anteversion · Two-dimensional imaging · Three-dimensional imaging · EOS imaging system 


\section{Introduction}

The goal of total hip arthroplasty (THA) is pain relief and restoration of normal daily activities. This can be achieved by proper implantation, restoring the offset and adequate soft tissue tensioning. Proper geometry for the reconstruction will also affect the forces around the hip, joint stability and long term survival of the implants [1-3]. The literature emphasizes that a decrease or increase more than $5 \mathrm{~mm}$ in offset (FO) can negatively affect the wear of the contact surfaces by increasing the joint reaction forces $[4,5]$.

The low-dose EOS imaging is an innovative slotscanning radiograph system providing valuable information in this specific field. The simultaneous capture of two orthogonal anteroposterior (AP) and lateral images (like standard X-rays) while the patient is standing, allows three-dimensional (3D) reconstruction in this functional position [6-12]. This technology is based on studies of gaseous detectors by Georges Charpak, who won the Nobel prize for physics in 1992. Besides the image resolution, the difference in accuracy might be related to the method of acquisition: conventional radiograph systems project the information on the patient's reference plane by means of a conical dimensional effect. The image quality decreases from the center toward the edges of the radiograph. EOS slot-scanning radiographs are always aligned with the detectors, thus enhancing image contrast. Due to the fan-beam geometry, the only alteration encountered during scanning is located along the horizontal axis. However, distortion is corrected by a new digital graduation executed by the visualization interface of the system. This image is reconstructed as if it had been acquired in the patient's reference plane by limiting the alteration of the patient's thickness instead of the source-to-detector distance. Therefore, the EOS system provides homogeneity on the whole radiograph. EOS was originally used in the perioperative and postoperative management of idiopathic scoliosis, considering the need for repeated radiographs and the importance of limiting the radiation dose. It will also provide us with the opportunity of the comparison of two2D and 3D data without additional imaging study. Some recent studies have shown that in comparison to the standard X-rays and computed tomography (CT) scans, EOS is accurate and reliable for the $2 \mathrm{D}$ and $3 \mathrm{D}$ radiographic assessment of the pelvis and lower limbs without significant inconvenience caused by the metallic artifacts of implants [13-16].

There are many studies published on the importance of offset restoration. To our knowledge, there is no study for accurate measurement of postoperative hip offset in functional standing position, comparing cemented and cementless femoral stems, with reliability and reproducibility. Most of the published data about the geometry of the THA are extracted from 2D X-rays and CT in the supine position [17-20]. In this study, we used both 2D and 3D X-rays taken by EOS system in the standing position to measure the postoperative hip offset among the two main stem types and compare it with the nonoperative hip as our reference hip. We set to find out how accurate the two dimensional AP X-ray was compared with the $3 \mathrm{D}$ imaging, and to assess the repeatability and reproducibility of the EOS imaging system. Our secondary goal was to see if we, orthopaedic surgeons, can restore the offset using the current techniques and implant designs for primary THA. An additional question was the analysis of the anteversion in the operative side compared with the non-implanted side and its potential impact on the offset.

\section{Methods and materials}

In this prospective study, we assessed the offset in 110 patients who underwent a unilateral THA by 15 surgeons with different experience levels and through different approaches. We used the EOS imaging system in our clinic for both preoperative and postoperative assessment of all orthopaedic patients (Figs. 1 and 2). We approached all the patients who had our inclusion criteria and described the purpose of the study, and their consent for participation was obtained. The patient demographics are presented in Table 1. The two groups (cemented and cementless stems) were homogeneous in terms of age, gender, height, and body mass index (BMI).

All patients had THA using one of the two major types of the femoral stems, non-modular cemented and uncemented for primary THA systems (Table 1). Neither of the patients had postoperative complications. The senior author evaluated the patients and reviewed their medical records before EOS imaging to make sure that they did not have any complications postoperatively and their contralateral hip was asymptomatic and without degenerative arthritis. Whenever the surgery was performed in another hospital, the original medical records, including the implant data, were obtained and reviewed in detail.

The offset was measured two- and three-dimensionally and compared between the operative and non-operative hips. The measurements were performed by two experienced operators separately and the results were evaluated for reliability and reproducibility. In addition the femoral anteversion was assessed in 3D images according to a validated protocol to determine if significant variations of this parameter could influence the results $[13,16]$.

\section{Statistical analysis}

Measurement of angular parameters was conducted by two independent operators (J.Y.L. and A.B...). Two successive 
Fig. 1 Biplanar acquisition of the pelvis in the standing position in two planes: anteroposterior and true lateral
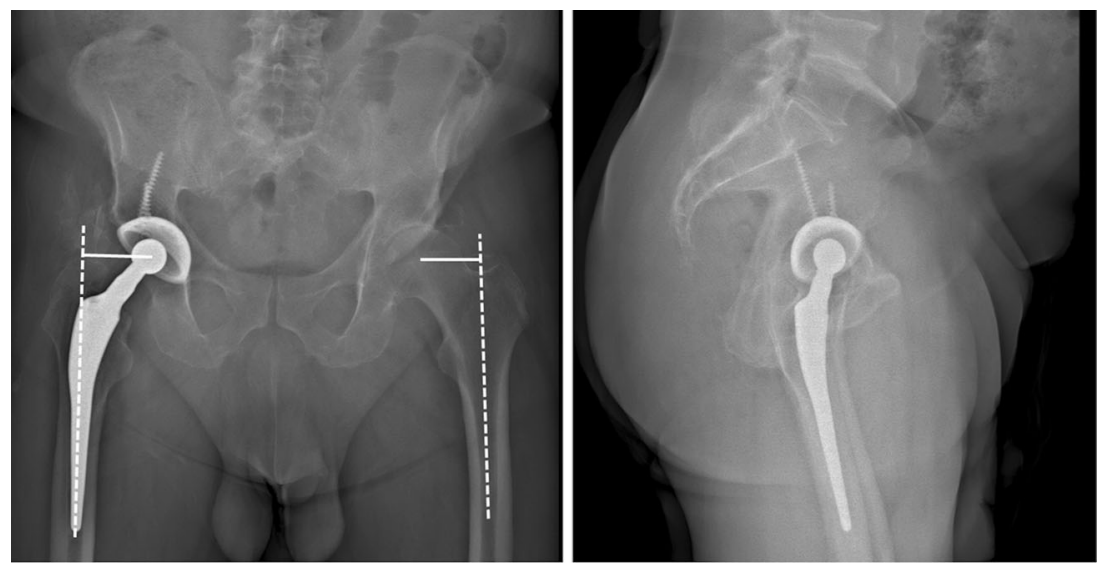

measurements were performed for each pelvic parameter by the operators. Measurements performed on 2D images were considered the reference measurements. The repeatability (intra-observer) and reproducibility (interobserver) of both imaging system measurements were independently calculated for 2D and 3D images [21-24].

First, the repeatability (intra-observer) and reproducibility (interobserver) of both offset measurements were independently calculated for "offset 2D" and "offset 3D". This analysis was inspired by the ISO 5725-2 standard [25]. This standard provides guidance for the determination of a $95 \%$ confidence interval for interobserver and/or intra-observer reproducibility. It uses a one-way random-effect model of analysis of variance (ANOVA). The calculated variables were: $\mathrm{S}_{\mathrm{L}}{ }^{2}$ was the estimated interobserver variance, $\mathrm{S}_{\mathrm{W}}{ }^{2}$ was the estimated intra-observer variance, $\mathrm{S}_{\mathrm{r}}{ }^{2}$ was the arithmetic mean of $\mathrm{S}_{\mathrm{W}}{ }^{2}$ representing the estimated repeatability variance, $\mathrm{S}_{\mathrm{R}}{ }^{2}$ was the estimated reproducibility variance with $\mathrm{S}_{\mathrm{R}}{ }^{2}=\mathrm{S}_{\mathrm{L}}{ }^{2}+\mathrm{S}_{\mathrm{r}}{ }^{2}$. Data were analyzed using Bland and Altman methods for agreement between the two measurements.
Fig. 2 Three-dimensional reconstruction of images in the standing position via STEREOS software
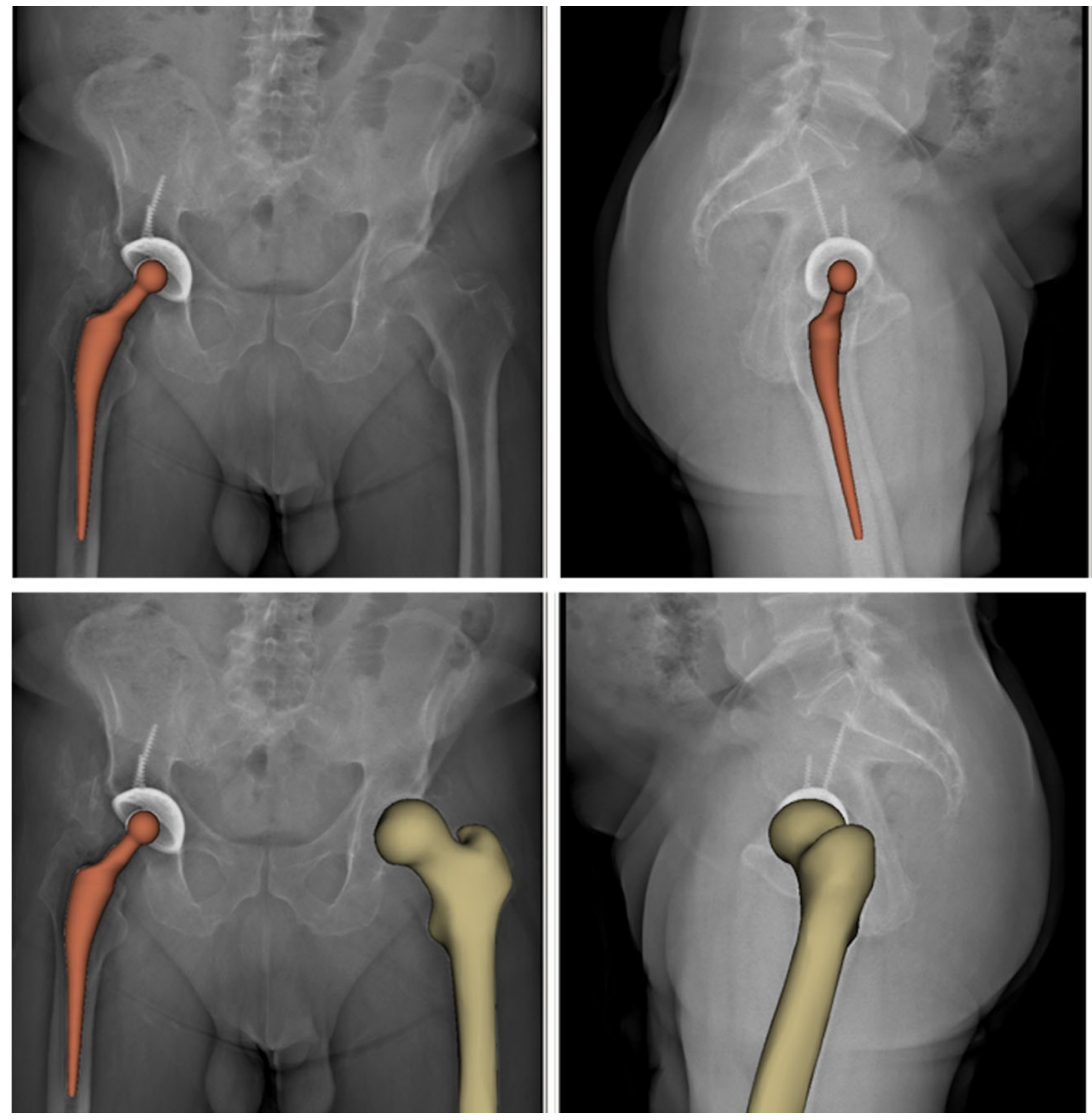
Table 1 Demographics and implant usage of the cohort

\begin{tabular}{ll}
\hline Variable & Results \\
\hline Age (years) & $64.8(29-90)$ \\
Gender & Female: 69 \\
& Male: 41 \\
Body mass index (BMI) & $26(22-31)$ \\
Time from surgery to evaluation & $4(1-8)$ \\
Type of fixation of acetabular implant & \\
Cemented & 20 \\
Cementless & 90 \\
Type of fixation of femoral implant & \\
Cemented & 48 \\
Cementless & 62 \\
Bearing type & \\
Ceramic or metal on polyethylene & 62 \\
Ceramic on ceramic & 30 \\
Metal on metal & 18 \\
Femoral implant neck angle & \\
$130-131^{\circ}$ & 84 (cemented 6) \\
$<130^{\circ}$ & 7 (cemented 3) \\
$135^{\circ}$ & 19 (cemented 11) \\
Approach & \\
Anterior & 12 \\
Anterolateral & \\
Posterior & \\
\hline &
\end{tabular}

Moreover, repeatability and reproducibility were assessed by calculating the interobserver and intra-observer intraclass correlation coefficient (ICC) and its $95 \%$ confidence interval. The ICC is defined as the ratio between explained variances (variance attributable to the cause of variation: observer factor; repetition of measurement) and overall variance (explained variance + error variance). Comparison of repeatability and reproducibility of each parameter was performed using the Fisher-Snedecor test for comparison of variances. Quantitative variables were described using the mean (M), the mean difference (d), the standard deviation (SD), the intraclass correlation coefficient (ICC) with its confidence interval (CI $95 \%$ ). We considered an intraclass correlation coefficient of $>0.90$ as high, between 0.80 and 0.90 as moderate, and $<0.80$ as insufficient.

Normal distribution of the values was checked by means of the Shapiro-Wilk normality test for each series of measurements. For data with normal distribution, paired Student $t$-test and independent sample $t$-test were used for analysis. For data without normal distribution, related samples Wilcoxon signed rank test and independent sample Mann-Whitney $U$ test were used for the analysis. Data were analyzed using the Medcalc software, version 11.3 and SPSS software (IBM) version 21.

\section{Results}

We compared the offset of the non-operative hips both twoand three-dimensionally in each patient. We also compared male and female patients separately as presented in Table 2. In the statistical analysis, the difference between the $2 \mathrm{D}$ and $3 \mathrm{D}$ offset measurements was significant for comparison of all the patients together $(p<0.001)$. This shows the insufficient accuracy of the $2 \mathrm{D}$ imaging studies in offset measurement. In the subgroup analysis, there was substantial difference in comparison of male and female patients separately $(p=0.02)$, or cemented $(p<0.01)$ and uncemented implants $(p<0.01)$ separately. Male patients had more offset in both native and implanted hip compared with female patients in both 2D $(p=0.02)$ and 3D evaluation $(p<0.001)$. We did not find any difference between the different surgical approaches for THA regarding the restoration of the offset.

We did not find any significant difference in femoral anteversion between the native and implanted hips in the 3D images $(p=0.6)$ and between male and female either for their native hips or after implantation. Overall, $42 \%$ of the patients had less than $5 \mathrm{~mm}$ difference in offset between the native and implanted hip joints two-dimensionally. In the 3D analysis, the offset of the implanted hip was within $5 \mathrm{~mm}$ of the native hip in $59 \%$ of the patients $(p<0.01)$.

In comparison of the cemented and uncemented technique, we did not find any significant difference regarding the offset restoration as presented in Table 2. Overall, $59.7 \%$ of the patients had less than $5 \mathrm{~mm}$ difference in offset between the native and uncemented THA joints three-dimensionally. In the $3 \mathrm{D}$ analysis, the offset of the cemented THA was within $5 \mathrm{~mm}$ of the native hip in $58 \%$ of the patients $(p=0.9)$. In regards to anteversion, no significant variations could be observed between cemented and cementless stems $(p=0.2)$. On these implanted hips the Pearson's correlation coefficient between stem anteversion and the difference between 2D and 3D offset measurements was 0.58 . No correlation with the operative approach could be demonstrated in this series.

The reliability analysis is presented in Table 3 and intraclass correlation coefficient is presented in Table 4, which shows high correlation in all measurements. Figure 3 shows the results of the Bland and Altman analysis for the agreement between the measurements which shows good agreement.

\section{Discussion}

In this study, we evaluated the offset restoration after THA in 110 patients using both 2D and 3D X-rays taken by the EOS system. We had two groups of patients based on the type of the femoral stem implanted: non-modular cemented and nonmodular cementless. To our knowledge, this is the first study of its kind. This study has its limitations. Because most of the 
Table 2 Joint offset and femoral anteversion in both native and implanted hips

\begin{tabular}{|c|c|c|c|c|c|c|c|c|}
\hline \multicolumn{9}{|c|}{ Two-dimensional offset } \\
\hline \multicolumn{3}{|l|}{ Male } & \multicolumn{3}{|l|}{ Female } & \multicolumn{3}{|l|}{ All } \\
\hline \multirow[t]{3}{*}{ Native hip } & Implanted & & Native hip & Implanted & & Native hip & Implanted & \\
\hline & Cemented & Uncemented & & Cemented & Uncemented & & Cemented & Uncemented \\
\hline & $4.37( \pm 0.44)$ & $4.44( \pm 0.84)$ & & $3.89( \pm 0.73)$ & $3.87( \pm 0.75)$ & & $4.07( \pm 0.68)$ & $4.08( \pm 0.83)$ \\
\hline $4.24( \pm 0.70)$ & $4.41( \pm 0.69)$ & & $3.77( \pm 0.64)$ & $3.88( \pm 0.74)$ & & $3.94( \pm 0.70)$ & $4.08( \pm 0.76)$ & \\
\hline $4.32( \pm 0.69)$ & & & $3.82( \pm 0.69)$ & & & $4.01( \pm 0.73)$ & & \\
\hline \multicolumn{9}{|c|}{ Three-dimensional offset } \\
\hline Male & & & Female & & & All & & \\
\hline \multirow[t]{3}{*}{ Native hip } & Implanted & & Native hip & Implanted & & Native hip & Implanted & \\
\hline & Cemented & Uncemented & & Cemented & Uncemented & & Cemented & Uncemented \\
\hline & $4.91( \pm 0.54)$ & $4.88( \pm 0.61)$ & & $4.01( \pm 0.62)$ & $4.27( \pm 0.61)$ & & $4.35( \pm 0.73)$ & $4.50( \pm 0.67)$ \\
\hline $4.65( \pm 0.53)$ & $4.90( \pm 0.57)$ & & $4.05( \pm 0.53)$ & $4.16( \pm 0.62)$ & & $4.27( \pm 0.60)$ & $4.43( \pm 0.70)$ & \\
\hline $4.77( \pm 0.56)$ & & & $4.10( \pm 0.58)$ & & & $4.35( \pm 0.66)$ & & \\
\hline \multicolumn{9}{|c|}{ Femoral anteversion } \\
\hline Male & & & Female & & & All & & \\
\hline \multirow[t]{3}{*}{ Native hip } & Implanted & & Native hip & Implanted & & Native hip & Implanted & \\
\hline & Cemented & Uncemented & & Cemented & Uncemented & & Cemented & Uncemented \\
\hline & $14.06( \pm 20.48)$ & $13.21( \pm 16.81)$ & & $12.92( \pm 15.85)$ & $15.02( \pm 12.63)$ & & $13.34( \pm 17.48)$ & $14.37( \pm 14.13)$ \\
\hline $11.94( \pm 11.46)$ & $13.60( \pm 18.30)$ & & $13.60( \pm 10.78)$ & $14.09( \pm 14.06)$ & & $13.00( \pm 11.00)$ & $13.91( \pm 15.64)$ & \\
\hline $12.77( \pm 15.181$ & & & $13.85( \pm 12.48)$ & & & $13.45( \pm 13.46)$ & & \\
\hline
\end{tabular}

Measurement units: for offset, centimeters ( $\mathrm{cm}$ ); for anteversion, degrees

patients were not assessed pre-operatively using the EOS system, we compared the operative and non-operative sides after arthroplasty. It is possible that the original offset of the operative hip before arthroplasty was not equal to the nonoperative hip. The surgeries were performed by 15 different surgeons in different hospitals: these surgeons had different experience level in THA and used different approaches. We believe this can also be one of the strengths of this study, because it gives us a reliable reference when assessing patients operated upon by other surgeons in the clinics. In addition, in this study we presented the repeatability and reproducibility of the results, which were not considered in many of the previously published studies.

The two main points that can be discussed in the light of this study are the improvement of offset measurement using $3 \mathrm{D}$ imaging rather than 2D AP X-ray, and the ability to restore the offset and anteversion in both cemented and uncemented THA.

There are three different imaging studies that can be used for offset measurement. Regular AP X-ray is available in all hospital and orthopaedic clinics. The 2D nature of plain X-ray, projection effect of the femoral anteversion and external rotational contracture of the degenerative hip result in limited accuracy of plain pelvic X-rays [19, 26, 27]. Plain AP pelvis X-rays could underestimate the offset value by $8-13 \%[17$, 28].

CT scan with 3D reconstruction is the other imaging study that can be used for measurement of the offset and hip geometry, but it exposes the patients to large radiation dose [29-31]. CT scan is supposed to be particularly accurate for the offset measures, but to our best knowledge, there is no study for repeatability and reproducibility of such measures. Because transverse scans pass through the femoral neck obliquely, accurate measurement of the offset might not be possible using CT scan. Also, thinner slices at the femoral neck region will be needed for improving the accuracy of measurements which will increase the radiation dose. In addition, another possible limitation is the difficulty to determine the true axis of the femoral shaft by scanning the proximal femur only if we want to reduce the radiation dose [32].
Table 3 Repeatability and reproducibility of offset measurements

\begin{tabular}{llllll}
\hline & \multicolumn{2}{l}{ Two-dimensional offset } & & \multicolumn{2}{l}{ Three-dimensional offset } \\
\cline { 2 - 3 } \cline { 5 - 6 } & Operative side & Non-operative side & & Operative side & Non-operative side \\
\hline Repeatability & 0.66 & 0.66 & & 0.46 & 0.46 \\
Reproducibility & 0.81 & 0.67 & & 0.47 & 0.56 \\
\hline
\end{tabular}


Table 4 Intraclass correlation coefficient (ICC)

\begin{tabular}{|c|c|c|c|c|}
\hline \multirow[t]{2}{*}{ ICC mean (CI $95 \%)$} & \multicolumn{2}{|c|}{ Two-dimensional offset } & \multicolumn{2}{|c|}{ Three-dimensional offset } \\
\hline & Operative side & Non-operative side & Operative side & Non-operative Side \\
\hline Repeatability & $0.81(0.72-0.87)$ & $0.87(0.82-0.91)$ & $0.82(0.74-0.88)$ & $0.85(0.79-0.90)$ \\
\hline Reproducibility & $0.84(0.79-0.89)$ & $0.90(0.86-0.93)$ & $0.81(0.75-0.87)$ & $0.85(0.80-0.89)$ \\
\hline
\end{tabular}

The ICC is defined as the ratio between explained variances (variance attributable to the cause of variation: observer factor; repetition of measurement) and overall variance (explained variance + error variance). We considered an intraclass correlation coefficient of $>0.90$ as high, between 0.80 and 0.90 as moderate, and $<0.80$ as insufficient

The full-body, 3D EOS system has less radiation compared with regular and digital X-ray and CT scan [33-35]. It provides the unique opportunity of studying the patient in functional standing and sitting position and assessing the relationship of pelvis and spine in functional body postures [36]. The EOS system has already been validated for the measurements $[13,15,16,34,35,37]$. Our results in regards to the native hips are in accordance with previously published data about femoral offset $[26,38]$. Our results for femoral version on the native side are also consistent with the literature reporting values from 13 to $23^{\circ}$ from $\mathrm{CT}$ scan and magnetic resonance imaging (MRI) measurements [39-43]. Two-dimensional measurements underestimate the offset for an average of $3.3 \mathrm{~mm}$ in native hips and $3.5 \mathrm{~mm}$ in implanted hips (2.8 $\mathrm{mm}$ for cemented stems and $4.2 \mathrm{~mm}$ for cementless stems). The main factor explaining this discrepancy is hip rotational positioning as the mean values for femoral anteversion are within the range previously published in the literature. In addition, no significant statistical correlation could be demonstrated between femoral version and 2D/3D offset discrepancies.

The importance of the offset restoration has been shown in the literature. It is well accepted that a restored offset results in a stable joint, better abductor function with less energy expenditure, less limp, equal leg length, and in the long term, less polyethylene wear $[2,3,17,44,45]$. This can be achieved by inferomedial positioning of the acetabular implant [46] and using a femoral implant with varus or longer neck; shifting the trunion medially is another alternative used in some implants [38].

The pre-operative templating is performed on $2 \mathrm{D}$ analog or digital X-rays in most orthopaedic centers. The low predictability of this approach for templating has been shown in previous publications [47-49]. This is more applicable to
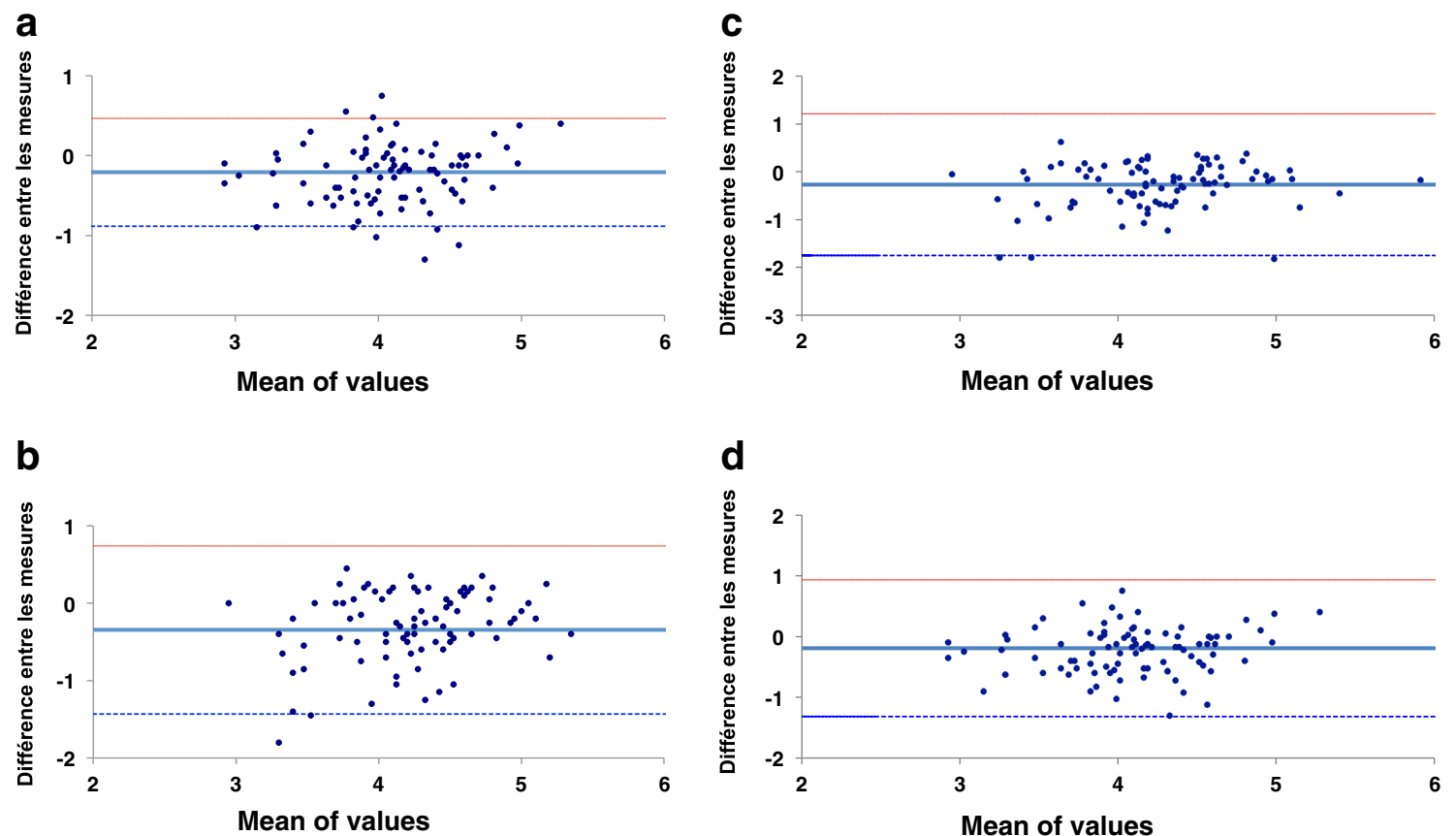

Fig. 3 a Repeatability of offset 2D/3D measurement in non-operative hips. b Repeatability of offset 2D/3D measurement in operative hips. c Reproducibility of offset 2D/3D measurement in operative hips. d

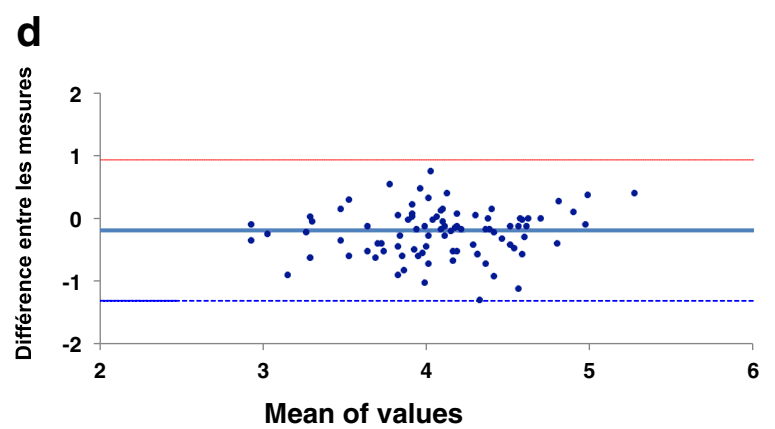

Reproducibility of offset 2D/3D measurement in non-operative hips. (Bland and Altman method) 
cementless stems because the final size of the stem is a compromise between the femoral bone density and the size of the femoral canal. In patients who are outliers in bone density and canal diameter, the surgeon may not be able to restore the offset using the non-modular implants according the preoperative templating. Many of the currently implanted femoral stems are non-modular and their offset changes with the size of the stem. The use of a head with longer or shorter neck size can also help with offset restoration to some extent, but it will affect the final limb length simultaneously. In the cemented stems, the orthopaedic surgeon has more freedom in adjusting the offset and implant position. By inserting the implant in more or less depth in the cement mantle, the surgeon can control the limb length and at the same time use an implant with a more or less varus neck-shaft angle and different length of the femoral neck.

The routine use of modular femoral prosthesis is a controversial topic among the adult reconstruction surgeons. These implants help the orthopaedic surgeons to restore the offset and version of the femoral neck easier compared with nonmodular implants, but with the cost of corrosion and failure at the modular sites [50-52]. The goal of THA is not to restore the offset with an accuracy of a $1 \mathrm{~mm}$ compared with the nonoperative side. The real problem is the detection of the patients who are outliers in their hip joint anatomy in terms of the shape of the proximal femur or version of the femoral neck during the pre-operative screening. These outliers might be good candidates for THA using modular femoral prosthesis instead of conventional non-modular implants. Unlike the EOS system, the pre-operative screening is not reliable by the standard 2D imaging studies and disproportionate in terms of price and irradiation for a CT scan. This study shows that conventional non-modular implants can restore joint offset and anteversion in the majority of cases in this series. Nevertheless the use of cemented stems failed to restore the femoral offset with more than $5 \mathrm{~mm}$ in $42 \%$ of patients. The results were quite the same for cementless stems with more than $5 \mathrm{~mm}$ imprecision in $40 \%$ of the cases. These results are consistent with previous estimated evaluation of outliers using conventional imaging technologies [45]. This study puts into perspective the use of the EOS imaging for pre-operative detection of outliers and the eventual planning of specific or modular implants in cases where sufficient restoration of the offset cannot be reasonably achieved by the conventional implants. In addition, this low-dose imaging technology can be used postoperatively to optimize the assessment of cases when offset failure is suspected.

\section{Conclusions}

There are many studies published on the importance of offset restoration. To our knowledge, there is no published study for accurate measurement of postoperative hip offset in functional standing position comparing two different types of nonmodular femoral stems, cemented and cementless, with reliability and reproducibility. The low-dose EOS technology provides new opportunities for planning the restoration of the femoral offset or to perform the post-operative control at the expense of much lower radiation dose than the CT scan and with a precision and reproducibility described on this unselected series of patients implanted with cemented or cementless prostheses. With the current implant design, the orthopaedic surgeons can restore the hip offset using both cemented or cementless implants. Detection of outliers who need modular prostheses for the primary THA could be one of the primary indications for the EOS imaging technique.

Conflict of interest The authors declare that they have no conflict of interest.

\section{References}

1. Charles MN, Bourne RB, Davey JR et al (2005) Soft-tissue balancing of the hip: the role of femoral offset restoration. Instr Course Lect 54: 131-141

2. Asayama I, Chamnongkich S, Simpson KJ et al (2005) Reconstructed hip joint position and abductor muscle strength after total hip arthroplasty. J Arthroplasty 20:414 420. doi:10.1016/j.arth. 2004.01.016

3. McGrory BJ, Morrey BF, Cahalan TD et al (1995) Effect of femoral offset on range of motion and abductor muscle strength after total hip arthroplasty. J Bone Joint Surg (Br) 77:865-869

4. Sakalkale DP, Sharkey PF, Eng K, et al. (2001) Effect of femoral component offset on polyethylene wear in total hip arthroplasty. Clin Orthop Relat Res 125-134

5. Schmidutz F, Beirer M, Weber P et al (2012) Biomechanical reconstruction of the hip: comparison between modular short-stem hip arthroplasty and conventional total hip arthroplasty. Int Orthop 36: 1341-1347. doi:10.1007/s00264-011-1477-2

6. Illés T, Somoskeöy S (2012) The EOS ${ }^{\mathrm{TM}}$ imaging system and its uses in daily orthopaedic practice. Int Orthop 36(7):1325-1331. doi:10. 1007/s00264-012-1512-y

7. Than P, Szuper K, Somoskeöy S, Warta V, Illés T (2012) Geometrical values of the normal and arthritic hip and knee detected with the EOS imaging system. Int Orthop 36(6):1291-1297. doi:10.1007/s00264011-1403-7

8. Chaibi Y, Cresson T, Aubert B et al (2012) Fast 3D reconstruction of the lower limb using a parametric model and statistical inferences and clinical measurements calculation from biplanar X-rays. Comput Methods Biomech Biomed Eng 15:457-466. doi:10.1080/ 10255842.2010.540758

9. Mitton D, Landry C, Véron S et al (2000) 3D reconstruction method from biplanar radiography using non-stereocorresponding points and elastic deformable meshes. Med Biol Eng Comput 38:133-139

10. Mitulescu A, Semaan I, de Guise JA et al (2001) Validation of the non-stereo corresponding points stereoradiographic 3D reconstruction technique. Med Biol Eng Comput 39:152-158

11. Le Bras A, Laporte S, Bousson V et al (2004) 3D reconstruction of the proximal femur with low-dose digital stereoradiography. Comput Aided Surg 9:51-57. doi:10.3109/10929080400018122 
12. Mitton D, Deschênes S, Laporte S et al (2006) 3D reconstruction of the pelvis from bi-planar radiography. Comput Methods Biomech Biomed Eng 9:1-5. doi:10.1080/10255840500521786

13. Buck FM, Guggenberger R, Koch PP, Pfirrmann CWA (2012) Femoral and tibial torsion measurements with 3D models based on low-dose biplanar radiographs in comparison with standard CT measurements. AJR Am J Roentgenol 199:W607-W612. doi:10.2214/ AJR.11.8295

14. Guenoun B, Zadegan F, Aim F et al (2012) Reliability of a new method for lower-extremity measurements based on stereoradiographic three-dimensional reconstruction. Orthop Traumatol Surg Res 98:506-513. doi:10.1016/j.otsr.2012.03.014

15. Lazennec J-Y, Rousseau MA, Rangel A et al (2011) Pelvis and total hip arthroplasty acetabular component orientations in sitting and standing positions: measurements reproductibility with EOS imaging system versus conventional radiographies. Orthop Traumatol Surg Res 97(4):3. doi:10.1016/j.otsr.2011.02.006

16. Folinais D, Thelen P, Delin C et al (2013) Measuring femoral and rotational alignment: EOS system versus computed tomography. Orthop Traumatol Surg Res 99(5):509-516. doi:10.1016/j.otsr.2012. 12.023

17. Merle C, Waldstein W, Pegg E et al (2012) Femoral offset is underestimated on anteroposterior radiographs of the pelvis but accurately assessed on anteroposterior radiographs of the hip. J Bone Joint Surg (Br) 94-B:477-482. doi:10.1302/0301-620X.94B4.28067

18. Renkawitz T, Schuster T, Grifka J et al (2010) Leg length and offset measures with a pinless femoral reference array during THA. Clin Orthop Relat Res 468:1862-1868. doi:10.1007/ s11999-009-1086-1

19. Sariali E, Mouttet A, Pasquier G et al (2009) Accuracy of reconstruction of the hip using computerised three-dimensional pre-operative planning and a cementless modular neck. J Bone Joint Surg (Br) 91: 333-340. doi:10.1302/0301-620X.91B3.21390

20. Sariali E, Mouttet A, Pasquier G, Durante E (2009) Threedimensional hip anatomy in osteoarthritis. Analysis of the femoral offset. J Arthroplasty 24:990-997. doi:10.1016/j.arth. 2008.04.031

21. Journois D (2004) Concordance between two variables: graphical approach (Bland and Altman's method). Rev Mal Respir 21:127-130

22. Rillardon L, Levassor N, Guigui P et al (2003) Validation of a tool to measure pelvic and spinal parameters of sagittal balance. Rev Chir Orthop Reparatrice Appar Mot 89:218-227

23. Fermanian J (2005) Validation of assessment scales in physical medicine and rehabilitation: how are psychometric properties determined? Ann Readapt Med Phys 48:281-287. doi:10.1016/j.annrmp. 2005.04.004

24. Fuhrman C, Chouaïd C (2004) Concordance between two variables: numerical approaches (qualitative observations - the kappa coefficient-; quantitative measures. Rev Mal Respir 21:123-125

25. International Organization for Standardization (1994) Application of statistics - accuracy (trueness and precision) of measurement methods and results - Part 2: Basic method for the determination of repeatability and reproducibility of a standard measurement method. ISO 5725-2:1994

26. Rubin PJ, Leyvraz PF, Aubaniac JM et al (1992) The morphology of the proximal femur. A three-dimensional radiographic analysis. $\mathrm{J}$ Bone Joint Surg (Br) 74:28-32

27. De Thomasson E, Mazel C, Guingand O, Terracher R (2002) Value of preoperative planning in total hip arthroplasty. Rev Chir Orthop Reparatrice Appar Mot 88:229-235

28. Pasquier G, Ducharne G, Ali ES et al (2010) Total hip arthroplasty offset measurement: is CT scan the most accurate option? Orthop Traumatol Surg Res 96:367-375. doi:10.1016/j.otsr.2010.02.006

29. Guyer B, Smith DS, Cady RB et al (1984) Dosimetry of computerized tomography in the evaluation of hip dysplasia. Skeletal Radiol $12: 123-127$
30. Jonsson A, Herrlin K, Jonsson K et al (1996) Radiation dose reduction in computed skeletal radiography. Effect on image quality. Acta Radiol 37:128-133

31. Lattanzi R, Baruffaldi F, Zannoni C, Viceconti M (2004) Specialised CT scan protocols for 3-D pre-operative planning of total hip replacement. Med Eng Phys 26:237-245. doi:10.1016/j.medengphy.2003. 11.008

32. Preininger B, Schmorl K, von Roth P et al (2012) Femoral offset (3D) in patients without osteoarthritis - index values from 200 hip joints. Open Orthop J 6:578-581. doi:10.2174/ 1874325001206010578

33. Deschênes S, Charron G, Beaudoin G et al (2010) Diagnostic imaging of spinal deformities: reducing patients radiation dose with a new slot-scanning X-ray imager. Spine 35:989-994. doi:10.1097/BRS. 0b013e3181bdcaa4

34. McKenna C, Wade R, Faria R et al (2012) EOS 2D/3D X-ray imaging system: a systematic review and economic evaluation. Health Technol Assess 16:1-188. doi:10.3310/hta16140

35. Wade R, Yang H, McKenna C et al (2013) A systematic review of the clinical effectiveness of EOS 2D/3D X-ray imaging system. Eur Spine J 22:296-304. doi:10.1007/s00586-012-2469-7

36. Lazennec J-Y, Brusson A, Rousseau M-A (2012) THA patients in standing and sitting positions: a prospective evaluation using the lowdose "full-body" EOS. Semin Arthroplast 23:220-225. doi:10.1053/ j.sart.2013.01.005

37. Wybier M, Bossard P (2012) Musculoskeletal imaging in progress: the EOS imaging system. Joint Bone Spine. doi:10.1016/j.jbspin. 2012.09.018

38. Bourne RB, Rorabeck CH (2002) Soft tissue balancing: the hip. J Arthroplasty 17:17-22. doi:10.1054/arth.2002.33263

39. Tomczak RJ, Guenther KP, Rieber A et al (1997) MR imaging measurement of the femoral antetorsional angle as a new technique: comparison with CT in children and adults. AJR Am J Roentgenol 168:791-794. doi:10.2214/ajr.168.3.9057536

40. Sugano N, Noble PC, Kamaric E (1998) A comparison of alternative methods of measuring femoral anteversion. J Comput Assist Tomogr 22:610-614

41. Weiner DS, Cook AJ, Hoyt WA, Oravec CE (1978) Computed tomography in the measurement of femoral anteversion. Orthopedics 1:299-306

42. Reikerås O, Bjerkreim I, Kolbenstvedt A (1983) Anteversion of the acetabulum and femoral neck in normals and in patients with osteoarthritis of the hip. Acta Orthop Scand 54:18-23

43. Murphy SB, Simon SR, Kijewski PK et al (1987) Femoral anteversion. J Bone Joint Surg Am 69:1169-1176

44. Sakai T, Sugano N, Ohzono K et al (2002) Femoral anteversion, femoral offset, and abductor lever arm after total hip arthroplasty using a modular femoral neck system. J Orthop Sci 7:62-67. doi:10. $1007 / \mathrm{s} 007760200010$

45. Lecerf G, Fessy MH, Philippot R et al (2009) Femoral offset: anatomical concept, definition, assessment, implications for preoperative templating and hip arthroplasty. Orthop Traumatol Surg Res 95:210 219. doi:10.1016/j.otsr.2009.03.010

46. Delp SL, Wixson RL, Komattu AV, Kocmond JH (1996) How superior placement of the joint center in hip arthroplasty affects the abductor muscles. Clin Orthop Relat Res 137-146

47. Gonzalez Della Valle A, Slullitel G, Piccaluga F, Salvati EA (2005) The precision and usefulness of preoperative planning for cemented and hybrid primary total hip arthroplasty. J Arthroplasty 20:51-58. doi:10.1016/j.arth.2004.04.016

48. Carter LW, Stovall DO, Young TR (1995) Determination of accuracy of preoperative templating of noncemented femoral prostheses. J Arthroplasty 10:507-513

49. Knight JL, Atwater RD (1992) Preoperative planning for total hip arthroplasty. Quantitating its utility and precision. J Arthroplasty 7(Suppl):403-409 
50. Sotereanos NG, Sauber TJ, Tupis TT (2013) Modular femoral neck fracture after primary total hip arthroplasty. J Arthroplasty 28:196.e7-196.e9. doi:10.1016/j.arth.2012.03. 050

51. Werner SD, Bono JV, Nandi S et al (2013) Adverse tissue reactions in modular exchangeable neck implants: a report of two cases. J Arthroplasty 28:543.e13-543.e15. doi:10.1016/j. arth.2012.07.026

52. Cooper HJ, Urban RM, Wixson RL et al (2013) Adverse local tissue reaction arising from corrosion at the femoral neck-body junction in a dual-taper stem with a cobalt-chromium modular neck. J Bone Joint Surg 95:865-872. doi:10.2106/JBJS.L.01042 